\title{
Wrapped Branes in Romans F(4) Gauged Supergravity
}

\section{Myungbo Shim*}

Department of Physics and Research Institute of Basic Science, Kyung Hee University,

Seoul 02447, Republic of Korea

E-mail: mbshim1213@khu.ac.kr

\section{Nakwoo Kim}

Department of Physics and Research Institute of Basic Science, Kyung Hee University, Seoul 02447, Republic of Korea

School of Physics, Korea Institute for Advanced Study,

Seoul 02445, Republic of Korea

E-mail: nkimekhu.ac.kr

We present an abridged version of our recent work [1]. In this talk, we analyze the spectrum of lower-dimensional anti-de Sitter (AdS) solutions in F(4) gauged supergravity in six dimensions. The configurations correspond to D4-branes partially wrapped on various supersymmetric cycles in special holonomy manifolds. As a comprehensive analysis, we re-visited and extended previous results. In detail, we study the cases of two, three, and four-dimensional supersymmetric cycles within Calabi-Yau threefold, fourfold, G2, and Spin(7) holonomy manifolds. Furthermore, we analyze the IR behavior and discuss the admissibility of the singular flows. We also report on non-supersymmetric AdS vacua and check their stability in the consistently truncated lowerdimensional effective action, using the Breitenlohner-Freedman bound.

Corfu Summer Institute 2019 "School and Workshops on Elementary Particle Physics and Gravity"

(CORFU2019)

31 August - 25 September 2019

Corfù, Greece

${ }^{*}$ Speaker. 


\section{Introduction}

In this talk, we address a brief summary of our results in [1] and their position in the context of searching for supersymmetric backgrounds of superstring/M-theory. One can consult [1] for more detailed treatments and the comprehensive list of the references for this subject.

Exploring the spectrum of supersymmetric anti-de Sitter solutions in String/M-theory is an intriguing enterprise due to its aesthetic geometric structure and AdS/CFT correspondence [2]. Maximally supersymmetric solutions, $\mathrm{AdS}_{4} \times S^{7}, \mathrm{AdS}_{5} \times S^{5}$, and $\mathrm{AdS}_{7} \times S^{4}$, are well-known, but we are eventually interested in the duality of more realistic gauge field theories, so constructing less-supersymmetric AdS backgrounds which do have 10 or 11 dimensional supergravity origin is a valuable endeavor. For this purpose one usually takes one of the following two approaches in search of supersymmetric AdS solutions. The first approach is to study the most general form of supersymmetric AdS solutions in the dimensions of interest, using the geometry of Killing spinors. One sometimes manages to find new solutions [3, 4], or discover interesting novel geometric structures e.g. in $[5,6,7,8,9]$. On the other hand, one can utilize lower-dimensional gauged supergravity models which are consistent truncation of 10 or 11 dimensional supergravities. Interesting AdS solutions may be obtained by studying the critical points of the scalar potential or considering spontaneous dimensional reduction by turning on various gauge fields, see e.g. [10].

In this talk, we are interested in wrapped brane configurations leading to various lower dimensional AdS vacua, in $D=6 F(4)$ gauged supergravity [11]. It has long been known that this particular theory is a consistent truncation of $D=10$ massive type IIA supergravity [12]. Note that it is also established more recently that this theory can be uplifted to IIB supergravity as well $[13,14,15]$. For definiteness we will consider in this talk uplifts to massive IIA ${ }^{1}$, where the relevant brane interpretation is as D4-branes in the presence of D8-branes. The AdS vacuum of $F(4)$ gauged supergravity has 16 supercharges, and the dual field theory is proposed to be a five-dimensional supersymmetric gauge theory with $U S p(2 N)$ gauge group and $N_{f}<8$ massless hypermultiplets in fundamental representation [16, 17, 18, 19], and see also this part of [1] for the references on the gravity side analysis. The duality was checked using localization formula and the results for entanglement entropy agree with $N^{5 / 2}$ scaling of degrees of freedom [20].

Among the AdS solutions from wrapped branes, $\mathrm{AdS}_{2}$ solutions can be interpreted as near horizon limit of magnetically charged black holes, and, on the field theory side, the entropy is associated with the topologically twisted index. For M2-brane theory, agreement between the two sides of AdS/CFT was shown in [21, 22]. As one tries to apply this relation to black holes in $F(4)$ gauged supergravity, the field theory computations in [23, 24, 25] and the supergravity side result match [26, 27, 28], only after a mistake in [29] is fixed: in this reference, an instanton-like contribution for four-cycles was overlooked, and a correct solution for Kähler 4-cycle was presented by M. Suh [26]. This realization has prompted our present work. We re-visit the construction of AdS solutions from wrapped branes in $F(4)$ gauged supergravity, and provide a list of supersymmetric and non-supersymmetric solutions. We fill other gaps in [29] by studying also the flows between $\mathrm{AdS}_{6}$ and configurations with lower-dimensional Lorentz symmetry, and study the admissibility IR singularities following the criteria of Maldacena-Nuñez [30] and Gubser [31]. We also provide the

\footnotetext{
${ }^{1}$ In IIB setting we have a network of D5 and NS5-branes preserving $(4+1)$-dimensional Lorentz symmetry.
} 
consistently truncated lower-dimensional actions, in the manner of [32], and study the fluctuation modes to see if they violate the Breitenlohner-Freedman bound [33] for stability.

\section{F(4) Gauged Supergravity}

\subsection{The Action and Its Relation to 10 Dimensions}

Let us first start by presenting the action of the bosonic sector for $D=6, F(4)$ gauged supergravity.

$$
\begin{aligned}
\mathscr{S}_{F(4)}= & \frac{1}{2 \kappa_{6}^{2}} \int d^{6} x \sqrt{-g}\left[\frac{1}{4} R-\frac{1}{2} \partial_{\mu} \phi \partial^{\mu} \phi+\frac{1}{8}\left(g^{2} e^{\sqrt{2} \phi}+4 g m e^{-\sqrt{2} \phi}-m^{2} e^{-3 \sqrt{2} \phi}\right)\right. \\
& -\frac{1}{4} e^{-\sqrt{2} \phi}\left(\mathscr{H}_{\mu v} \mathscr{H}^{\mu v}+F_{\mu \nu}^{I} F^{I \mu v}\right)-\frac{1}{12} e^{2 \sqrt{2} \phi} G_{\mu v \rho} G^{\mu v \rho} \\
& \left.-\frac{1}{8} \varepsilon^{\mu \nu \rho \sigma \tau \kappa} B_{\mu v}\left(\mathscr{F}_{\rho \sigma} \mathscr{F}_{\tau \kappa}+m B_{\rho \sigma} \mathscr{F}_{\tau \kappa}+\frac{1}{3} m^{2} B_{\rho \sigma} B_{\tau \kappa}+F_{\rho \sigma}^{I} F_{\tau \kappa}^{I}\right)\right] .
\end{aligned}
$$

The action as it stands includes gravity via metric $g_{\mu \nu}$, a two-form tensor field $B$ with field strength $G=d B$, a triplet of $S U(2)$ gauge fields $A^{I}$, a $U(1)$ vector $\mathscr{A}$, and a real scalar field $\phi . \mathscr{H}$ is a combination of the field strength $\mathscr{F}=d \mathscr{A}$ and two-form tensor field, namely $\mathscr{H}=\mathscr{F}+m B$. Note that the total number of on-shell bosonic degrees of freedom is 32 . We have two coupling constants, $g$ and $m$, in addition to Newton constant $\kappa_{6}$.

When we uplift this system to $D=10$ massive IIA supergravity, we have $m_{10 d}=\sqrt{2} m$ in the convention of e.g. [12, 29]. We note that there exist alternative embeddings into type IIB theory which was recently found in $[13,14]^{2}$, and also into the exceptional field theory formalism $[34,15]$. This theory allows a supersymmetric $\mathrm{AdS}_{6}$ solution when $e^{-2 \sqrt{2} \phi}=g / 3 m$ and all other bosonic (and also fermionic) fields are trivial. When uplifted, it is a 1/2-BPS configuration of IIA/IIB supergravity in $D=10$. In the convention we adopt, the radius of AdS space is $L_{A d S_{6}}=$ $3 \sqrt{2}\left(3 m g^{3}\right)^{-1 / 4}$, or $3 \sqrt{2} / g$ when we substitute $m=g / 3$ as a convenient choice for the theory which can be uplifted in IIA/IIB.

\subsection{A Survey of Wrapped Brane Solutions}

In this talk, we are interested in a specific type of classical solutions: in particular, we have lower-dimensional anti-de Sitter spaces in mind. This type of solutions were known as "magnetovacs" before the advent of string duality and D-branes [10]. Thanks to a seminal paper of Maldacena-Nuñez [35], and the extension to higher-dimensional cycles [36, 37, 38], these solutions are nowadays commonly referred to "wrapped-brane" solutions. The way how to produce such non-trivial solutions is as follows. We assume that part of the space is Einstein (which corresponds to supersymmetric cycles), and turn on gauge connection and impose Killing spinor projection rules so that the contributions of spin connection and gauge connection exactly cancel, at least along the cycle directions. This is the manifestation of topological twisting (via cancelling the spin connection, we effectively turn a spinor into a scalar). Depending on the concrete choice of gauge connection, we deal with different kinds of special holonomy manifolds and supersymmetric cycles thereof.

\footnotetext{
${ }^{2}$ One can find out other references on the embedding of the solutions in [1]
} 


\begin{tabular}{cccc}
\hline Cycles & $\mathscr{F}$ & $F_{\mu \nu}^{\hat{I}}$ & $B_{\mu v}$ \\
\hline 2-Cycles & 0 & $F_{45}^{\hat{3}}=\frac{k g}{g} e^{-2 \lambda}$ & 0 \\
3-Cycles & 0 & $F_{\text {non-zero }}^{\hat{I}}=\frac{k \zeta_{l}}{2 g} e^{-2 \lambda}$ & 0 \\
Cayley 4-Cycles & 0 & $F_{\text {non-zero }}^{\hat{I}}=\frac{k \zeta_{I}}{3 g} e^{-2 \lambda}$ & $B_{01}=-\frac{2}{3 m^{2} g^{2}} e^{\sqrt{2} \phi-4 \lambda}$ \\
Kähler 4-Cycles & 0 & $F_{23}^{\hat{3}}=F_{45}^{\hat{3}}=\frac{k \zeta}{g} e^{-2 \lambda}$ & $B_{01}=-\frac{2}{m^{2} g^{2}} e^{\sqrt{2} \phi-4 \lambda}$ \\
Kähler $\Sigma_{\mathfrak{g}_{1}} \times \Sigma_{\mathfrak{g}_{2}}$ & 0 & $F_{23}^{\hat{3}}=\frac{k_{1} \zeta}{g} e^{-2 \lambda_{1}}, F_{45}^{\hat{3}}=\frac{k_{2} \zeta}{g} e^{-2 \lambda_{2}}$ & $B_{01}=-2 \frac{k_{1} k_{2}}{m^{2} g^{2}} e^{\sqrt{2} \phi-2\left(\lambda_{1}+\lambda_{2}\right)}$ \\
\hline
\end{tabular}

Table 1: The ansatz for gauge fields in orthonormal bases for each case. Non-vanishing components are easily read off from the twisting condition. $\zeta_{(I)}$ is \pm 1 , representing the choice of orientation of wrapped branes. It is also constrained by $\zeta_{1} \zeta_{2} \zeta_{3}=1 . k= \pm 1$ gives the sign of scalar curvature of the supersymmetric cycles.

More concretely, the metric ansatz goes like

$$
d s_{6}^{2}=e^{2 f(r)}\left(-d t^{2}+d r^{2}+\sum_{\alpha=1}^{4-d} d x_{\alpha}^{2}\right)+\sum_{i} e^{2 \lambda_{i}(r)} d s_{\mathscr{M}_{i, d}}^{2} .
$$

On the right-hand-side, the part with scale factor $e^{2 f}$ contains the reduced worldvolume (after wrapping) and the "holographic" coordinate $r$. Then the latter part with scale factors $e^{2 \lambda_{i}}$ denotes the "supersymmetric cycle". For our purposes here, this part is either a single Einstein space or a sum of two Einstein spaces up to scale factors which is a function of $r$ only. The cycle part will be chosen as (sum of) constant curvature spaces, e.g. the sphere $S^{d}$, the complex projective manifold $\mathbb{C P}^{n}$, and their negatively-curved cousins such as the hyperbolic manifold and the Bergman space for concreteness. Then we turn on magnetic field for the $S U(2)$ part of the vector fields. The point is to make sure the effect of spin connection and gauge connection cancel along the cycle directions for spinors satisfying certain projection rules. We have checked that all solutions in previous works $[29,26]^{3}$ can be obtained from these BPS equations.

Explicit ansatz for each case is summarized in Table 1, and the properties of the solutions are provided in Table 2.

\section{Holographic RG Flows for Supersymmetric Solutions}

\subsection{2 and 3 Cycles}

Let us start with the cases of 2- and 3-cycles. They are relatively simple since the tensor field vanishes, so we treat them collectively. For the former the $S O(2)$ spin connection is identified with $U(1) \subset S U(2)$, and for the latter we identify $S O(3)$ spin connection with the entire $S U(2)$ gauge connection. In IIA description, 2-cycle is inside Calabi-Yau threefold, and for the latter we have associative 3-cycles inside $G_{2}$ holonomy manifold. The setup for vector fields and the projection rule is as given in the table below. Note that except for AdS fixed point solutions, we have an additional projection involving the radial direction. However, AdS fixed point solutions do not

\footnotetext{
${ }^{3}$ The correct solutions for 2-, 3-cycles and the solutions for 4-cycles are, respectively, discovered in the former and the latter.
} 


\begin{tabular}{ccccc}
\hline Cycles & $k$ & $\begin{array}{c}\text { BPS } \\
\text { solution }\end{array}$ & $\begin{array}{c}\text { Non-BPS } \\
\text { solution }\end{array}$ & $\begin{array}{c}\text { Does non-BPS solution } \\
\text { violate the BF Bound? }\end{array}$ \\
\hline \multirow{2}{*}{ 2-Cycles } & 1 & $\mathrm{X}$ & $\mathrm{X}$ & - \\
& -1 & $\mathrm{O}$ & $\mathrm{O}$ & Yes \\
\hline \multirow{2}{*}{ 3-Cycles } & 1 & $\mathrm{X}$ & $\mathrm{X}$ & - \\
& -1 & $\mathrm{O}$ & $\mathrm{O}$ & Yes \\
\hline $\mathbb{H}_{2} \times \mathbb{H}_{2}$ & $(-1,-1)$ & $\mathrm{O}$ & $\mathrm{X}$ & - \\
\hline$S^{2} \times S^{2}$ & $(1,1)$ & $\mathrm{X}$ & $\mathrm{O}$ & No \\
\hline$S^{2} \times \mathbb{H}_{2}$ & $(1,-1)$ & $\mathrm{X}$ & $\mathrm{X}$ & - \\
\hline \multirow{2}{*}{ Kähler 4-Cycles } & 1 & $\mathrm{X}$ & $\mathrm{O}$ & No \\
\hline \multirow{2}{*}{ Cayley 4-Cycles } & -1 & $\mathrm{O}$ & $\mathrm{X}$ & - \\
\hline
\end{tabular}

Table 2: A summary of existence of wrapped brane solutions in $F(4)$ gauged supergravity.

require such an extra condition, hence exhibit supersymmetry enhancement. Here $T^{\hat{i}}=i \sigma^{i} / 2$ is $S U$ (2) generator (anti-Hermitian), and $\gamma^{a}$ are $D=6$ gamma matrices. Parameters $\zeta, \zeta_{i}$ are \pm 1 and represent the choice of BPS conditions, and in particular $\zeta_{1} \zeta_{2} \zeta_{3}=1{ }^{4}$

\begin{tabular}{|c|ll|}
\hline 2-cycles & $\omega_{45}=\zeta_{g} A^{\hat{3}}$, & $T^{\hat{3}} \varepsilon=-\frac{1}{2} \zeta \gamma^{45} \varepsilon$ \\
\hline \multirow{3}{*}{ 3-cycles } & $\omega_{34}=\zeta_{1} g A^{\hat{1}}$, & $T^{\hat{1}} \varepsilon=-\frac{1}{2} \zeta_{1} \gamma^{34} \varepsilon$ \\
& $\omega_{53}=\zeta_{2} g A^{\hat{2}}$, & $T^{\hat{2}} \varepsilon=-\frac{1}{2} \zeta_{2} \gamma^{53} \varepsilon$ \\
& $\omega_{45}=\zeta_{3} g A^{\hat{3}}$, & $T^{\hat{3}} \varepsilon=-\frac{1}{2} \zeta_{3} \gamma^{45} \varepsilon$ \\
\hline
\end{tabular}

We set the tensor field to zero, and there are three functions we need to determine: $f, \lambda, \phi$. BPS equations are given below, where $d=2,3$ and denote the dimensionality of the supersymmetric cycles. Note that $k=1$ is for the sphere and $k=-1$ is for the hyperbolic spaces, with constant curvature.

$$
\begin{aligned}
f^{\prime} e^{-f} & =-\frac{1}{4 \sqrt{2}}\left[g e^{\frac{1}{\sqrt{2}} \phi}+m e^{-\frac{3}{\sqrt{2}} \phi}-\frac{d k}{g} e^{-\frac{1}{\sqrt{2}} \phi-2 \lambda(r)}\right], \\
\lambda^{\prime} e^{-f} & =-\frac{1}{4 \sqrt{2}}\left[g e^{\frac{1}{\sqrt{2}} \phi}+m e^{-\frac{3}{\sqrt{2}} \phi}+\frac{(8-d) k}{g} e^{-\frac{1}{\sqrt{2}} \phi-2 \lambda(r)}\right], \\
\frac{\phi^{\prime}}{\sqrt{2}} e^{-f} & =-\frac{1}{4 \sqrt{2}}\left[-g e^{\frac{1}{\sqrt{2}} \phi}+3 m e^{-\frac{3}{\sqrt{2}} \phi}+\frac{d k}{g} e^{-\frac{1}{\sqrt{2}} \phi-2 \lambda(r)}\right] .
\end{aligned}
$$

To facilitate the analysis, we find it convenient to introduce new variables as follows, as advocated in [37]. The BPS equations take a bit simpler form in terms of $x:=e^{2 \lambda-\sqrt{2} \phi}$ and $F:=x e^{2 \sqrt{2} \phi}$,

$$
\frac{d F}{d x}=\frac{2 F[2 k+m g x]}{x\left(g^{2} F-m g x+(4-d) k\right)} .
$$

\footnotetext{
${ }^{4}$ We point out that it is obviously inconsistent to set $\zeta_{1}=\zeta_{2}=\zeta_{3}$, and correct an error in eq.(4.13) of Ref.[26].
} 
We find there exist AdS fixed points for $k=-1$ :

$$
\begin{array}{lll}
d=2: & F=4 / g^{2}, & x=2 / g m, \\
d=3: & F=3 / g^{2}, & x=2 / g m .
\end{array}
$$
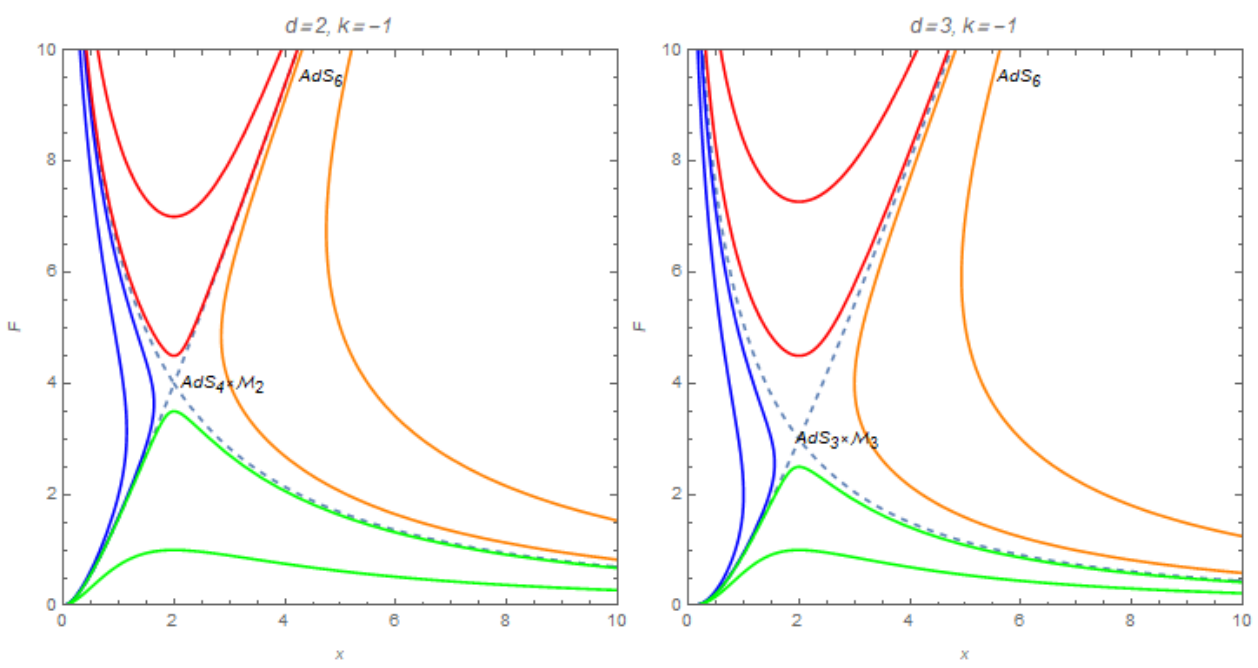

Figure 1: Flow Diagrams for negatively curved 2 and 3 cycles $(k=-1)$

We have not managed to integrate Eq.(3.2) explicitly. However, in UV regime where $g_{t t}$ becomes large and the metric asymptotes to $\mathrm{AdS}_{6}$, one easily sees that the solution can be written in a series expansion form,

$$
F=\frac{3 m}{g} x+\frac{3 d k}{g^{2}}+\frac{1}{g^{2}} \sum_{n=1}^{\infty} \frac{c_{n}}{(m g x)^{\frac{n}{2}}} .
$$

Here $c_{1}$ is an integration constant which parametrizes different solutions, and $c_{n}(n>1)$ can be determined recursively in terms of $c_{1}$. Numerically we find that the flows to $\operatorname{Ad}_{4}(d=2)$ and $A d S_{3}(d=3)$ correspond to $c_{1}=9.1296$ and $c_{1}=13.951$ respectively.

Other than the flows to AdS fixed points, there are three different kinds of "IR" singularities, according to Figure 1.

It turns out that all the singularities are good under the criterion of Ref.[30], which instructs us to study the behavior of $g_{t t}$. On the other hand, under the criterion of Ref.[31], where it was suggested we check the behavior of scalar potential, the singularities with small $F$ are bad. It turns out that the latter criterion is more strict for the solutions at hand. When $k=1$, there is no fixed point, but there are flows to good IR singularities.

Below is a summary of the analyses on the type of singularities. We see that the classification is not clear-cut, in particular for solutions with $F \rightarrow 0$ and $x \rightarrow \infty$.

\begin{tabular}{|c|c|c|c|c|c|c|}
\hline$k$ & $x$ & $F$ & $e^{2 f}$ & $\left|g_{t t}^{10 d}\right|$ & $V(\phi)$ & Type \\
\hline \pm 1 & $\infty$ & 0 & 0 & 0 & $\infty(\mathrm{bad})$ & - \\
\pm 1 & 0 & 0 & $\infty$ & $\infty$ & $\infty(\mathrm{bad})$ & Bad \\
-1 & 0 & $\infty$ & 0 & 0 & $-\infty$ & Good \\
\hline
\end{tabular}



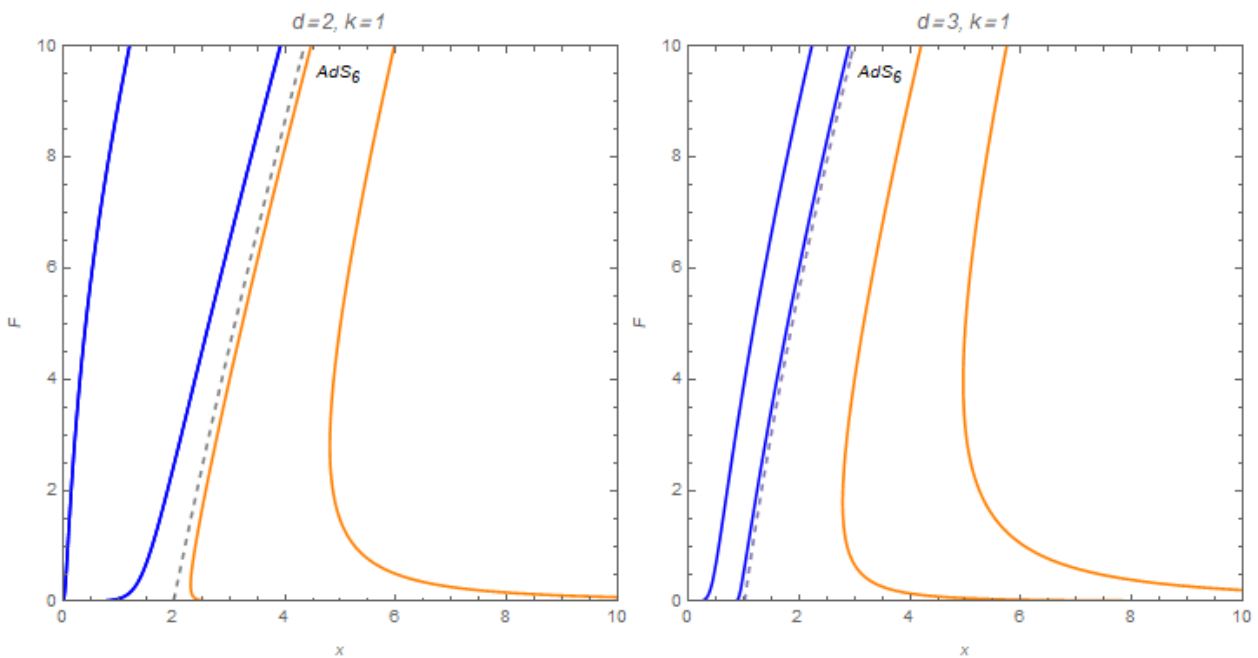

Figure 2: Flow Diagrams for positively curved 2 and 3 cycles, $k=1$

\subsection{4-Cycles}

Now let us turn to 4-cycles. There are two choices for partial twisting now: one is Kähler 4-cycle inside a Calabi-Yau 4-manifold, and the other is Cayley 4-cycle inside Spin(7) holonomy manifold. For the former, we identify the $U(1)$ part of spin connection with $U(1) \subset S U(2)$ part of the gauge connections. And for the latter, we set the $S U(2)$ gauge fields to the self-dual part of the spin connection. The BPS conditions are given as below.

\begin{tabular}{|c|ll|}
\hline Kähler 4-cycle & $\omega_{23} \pm \omega_{45}=g \zeta A^{\hat{3}}$, & $\frac{1}{2} \gamma_{23} \varepsilon= \pm \frac{1}{2} \gamma_{45} \varepsilon=-\zeta T^{\hat{3}} \varepsilon$ \\
\hline Cayley cycle & $\omega_{23} \pm \omega_{45}=g \zeta_{1} A^{\hat{1}}$, & $\frac{1}{2} \gamma_{23} \varepsilon= \pm \frac{1}{2} \gamma_{45} \varepsilon=-\zeta_{1} T^{\hat{1}} \varepsilon$ \\
$\gamma_{i j}^{\mp} \varepsilon=0$, & $\omega_{42} \pm \omega_{35}=g \zeta_{2} A^{\hat{2}}$, & $\frac{1}{2} \gamma_{42} \varepsilon= \pm \frac{1}{2} \gamma_{35} \varepsilon=-\zeta_{2} T^{\hat{2}} \varepsilon$ \\
$(i, j=2, \cdots, 5)$ & $\omega_{34} \pm \omega_{52}=g \zeta_{3} A^{\hat{3}}$, & $\frac{1}{2} \gamma_{34} \varepsilon= \pm \frac{1}{2} \gamma_{52} \varepsilon=-\zeta_{3} T^{\hat{3}} \varepsilon$ \\
\hline
\end{tabular}

In the above $\zeta, \zeta_{i}$ are \pm 1 , and $\zeta_{i}$ are constrained by $\zeta_{1} \zeta_{2} \zeta_{3}=1$. The associated BPS equations are presented below, where a constant $\Upsilon$ denotes non-vanishing instanton density and takes different values for Kähler $\left(\Upsilon=-\frac{1}{\sqrt{2} g^{2} m}\right)$ and Cayley 4-cycles $\left(\Upsilon=-\frac{1}{3 \sqrt{2} g^{2} m}\right)$.

$$
\begin{aligned}
f^{\prime} e^{-f} & =-\frac{1}{4 \sqrt{2}}\left[g e^{\frac{1}{\sqrt{2}} \phi}+m e^{-\frac{3}{\sqrt{2}} \phi}-\frac{4 k}{g} e^{-\frac{1}{\sqrt{2}} \phi-2 \lambda(r)}\right]+3 \Upsilon e^{\frac{1}{\sqrt{2}} \phi-4 \lambda(r)}, \\
\lambda^{\prime} e^{-f} & =-\frac{1}{4 \sqrt{2}}\left[g e^{\frac{1}{\sqrt{2}} \phi}+m e^{-\frac{3}{\sqrt{2}} \phi}+\frac{4 k}{g} e^{-\frac{1}{\sqrt{2}} \phi-2 \lambda(r)}\right]-\Upsilon e^{\frac{1}{\sqrt{2}} \phi-4 \lambda(r)}, \\
\frac{\phi^{\prime}}{\sqrt{2}} e^{-f} & =-\frac{1}{4 \sqrt{2}}\left[-g e^{\frac{1}{\sqrt{2}} \phi}+3 m e^{-\frac{3}{\sqrt{2}} \phi}+\frac{4 k}{g} e^{-\frac{1}{\sqrt{2}} \phi-2 \lambda(r)}\right]+\Upsilon e^{\frac{1}{\sqrt{2}} \phi-4 \lambda(r)} .
\end{aligned}
$$

When we adopt new variables $x:=e^{2 \lambda-\sqrt{2} \phi}, F:=e^{2 \sqrt{2} \phi} x$, the flow equations are reduced to

$$
\frac{d F}{d x}=\frac{F(2 m g x+4 k)}{x\left(g^{2} F-m g x\right)+4 \sqrt{2} g \Upsilon} .
$$

For a Kähler 4-cycle, we have an $\mathrm{AdS}_{2}$ fixed point when the cycle is negatively curved $(k=-1)$ at $F=4 / g^{2}, x=2 / g m$. On the other hand, for a Cayley 4-cycle, we have a supersymmetric fixed 
point when $k=-1, F=8 / 3 g^{2}, x=2 / g m$. Series expansion solutions can be also easily worked out, and we have

$$
F=\frac{3 m}{g} x+\frac{12 k}{g^{2}}+\frac{1}{g^{2}} \sum_{n=1}^{\infty} \frac{c_{n}}{(m g x)^{\frac{n}{2}}}
$$

Just like 2- and 3-cycle cases, $c_{n}(n>1)$ can be determined recursively in terms of $c_{1}$ when we substitute this expression into Eq.(3.7). Numerically we find that the flow to $\mathrm{AdS}_{2}$ corresponds to $c_{1}=23.538$ for Kähler case, and $c_{1}=19.7959$ for Cayley case.
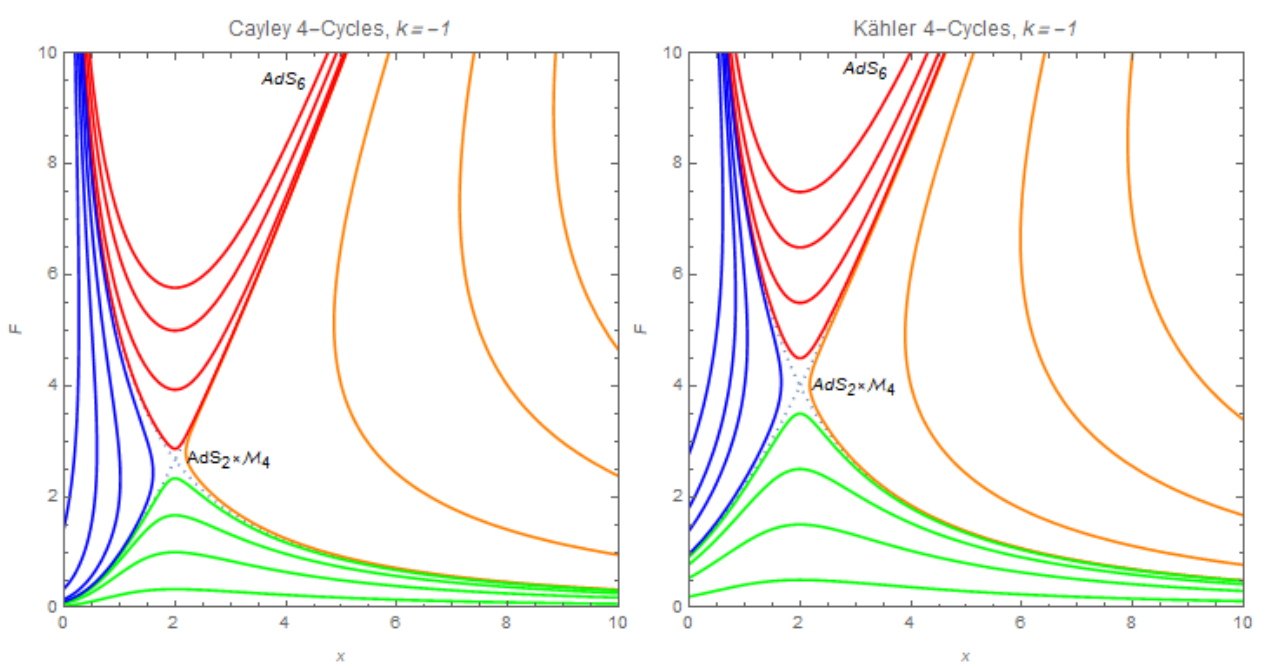

Figure 3: Flow Diagrams for negatively curved Cayley and Kähler 4-cycles, $k=-1$

Qualitatively speaking, when we analyze the UV asymptotics of $F(x)$, we notice that its behavior is similar to that of Eq.(3.5) with substitution $d=4$. In IR, both good and bad type singularities exist under the criterion in [30], but there is no good singularity according to the criterion in [31]. The flow diagrams and the types of singularities with respect to corresponding limit are summarized in Fig. 3, Fig. 4 and the table below.

\begin{tabular}{|c|c|c|c|c|c|c|}
\hline$k$ & $x$ & $F$ & $e^{2 f}$ & $\left|g_{t t}^{10 d}\right|$ & $V(\phi)$ & Type \\
\hline \pm 1 & $\infty$ & 0 & 0 & 0 & $\infty(\mathrm{Bad})$ & - \\
\pm 1 & 0 & 0 & $\infty$ & $\infty$ & $\infty(\mathrm{Bad})$ & $\mathrm{Bad}$ \\
\pm 1 & 0 & Finite & $\infty$ & $\infty$ & $\infty(\mathrm{Bad})$ & $\mathrm{Bad}$ \\
-1 & 0 & $\infty$ & 0 & 0 & $\infty(\mathrm{Bad})$ & - \\
\hline
\end{tabular}

\subsection{Kähler 4-Cycles as a Product of Two Riemann Surfaces}

For the Kähler 4-cycle case, in fact, one may consider a generalization where it is a direct product of two Riemann surfaces and allow different radii. The twisting and projection rules are

$$
\omega^{23}+\omega^{45}=g \zeta A^{\hat{3}}, \quad \frac{1}{2} \gamma_{23} \varepsilon=\frac{1}{2} \gamma_{45} \varepsilon=-\zeta T^{\hat{3}} \varepsilon .
$$



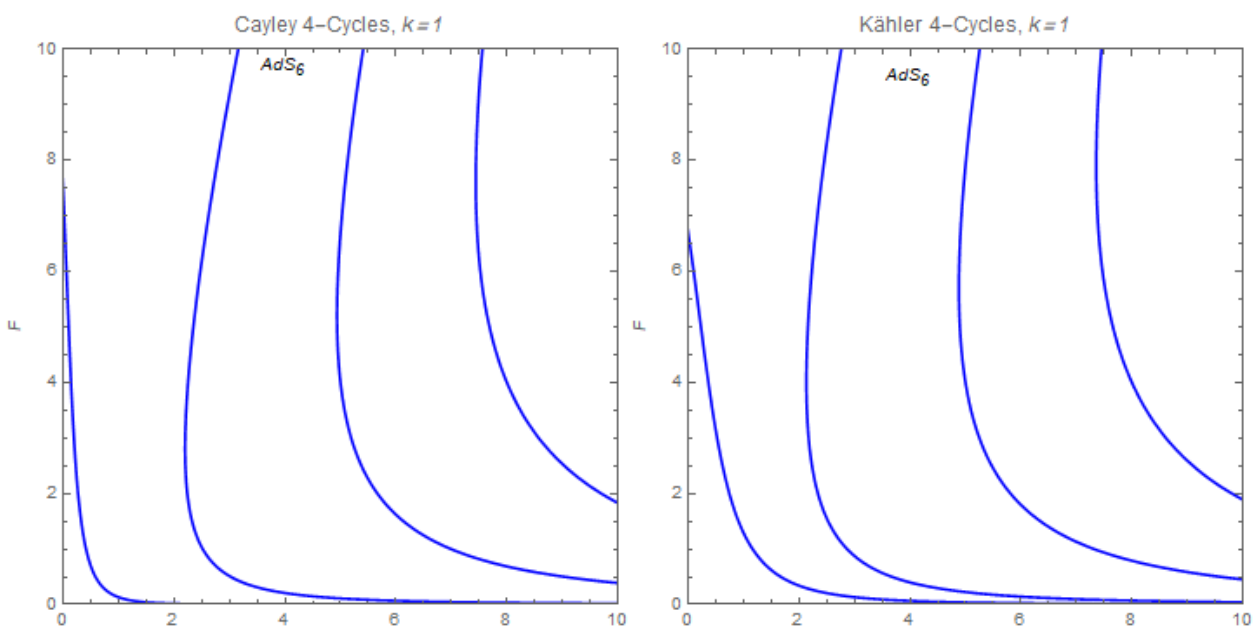

Figure 4: Flow Diagrams for positively curved Cayley and Kähler 4-cycles, $k=1$

Now we have four lines of BPS equations, as below. Note that they reduce to the previous BPS equations for 4-cycles Eq.(3.6) through identification, $\lambda_{1}=\lambda_{2}, k_{1}=k_{2}$, and setting $\Upsilon=-\frac{1}{\sqrt{2} g^{2} m}$.

$$
\begin{aligned}
f^{\prime} e^{-f} & =-\frac{1}{4 \sqrt{2}}\left[g e^{\frac{1}{\sqrt{2}} \phi}+m e^{-\frac{3}{\sqrt{2}} \phi}-\frac{2}{g} e^{-\frac{1}{\sqrt{2}} \phi}\left(k_{1} e^{-2 \lambda_{1}(r)}+k_{2} e^{-2 \lambda_{2}(r)}\right)\right]-3 \Upsilon e^{\frac{1}{\sqrt{2}} \phi-2 \lambda_{1}(r)-2 \lambda_{2}(r)}, \\
\lambda_{1}^{\prime} e^{-f} & =-\frac{1}{4 \sqrt{2}}\left[g e^{\frac{1}{\sqrt{2}} \phi}+m e^{-\frac{3}{\sqrt{2}} \phi}+\frac{2}{g} e^{-\frac{1}{\sqrt{2}} \phi}\left(3 k_{1} e^{-2 \lambda_{1}(r)}-k_{2} e^{-2 \lambda_{2}(r)}\right)\right]+\Upsilon e^{\frac{1}{\sqrt{2}} \phi-2 \lambda_{1}(r)-2 \lambda_{2}(r)}, \\
\lambda_{2}^{\prime} e^{-f} & =-\frac{1}{4 \sqrt{2}}\left[g e^{\frac{1}{\sqrt{2}} \phi}+m e^{-\frac{3}{\sqrt{2}} \phi}+\frac{2}{g} e^{-\frac{1}{\sqrt{2}} \phi}\left(-k_{1} e^{-2 \lambda_{1}(r)}+3 k_{2} e^{-2 \lambda_{2}(r)}\right)\right]+\Upsilon e^{\frac{1}{\sqrt{2}} \phi-2 \lambda_{1}(r)-2 \lambda_{2}(r)}, \\
\frac{\phi^{\prime}}{\sqrt{2}} e^{-f} & =-\frac{1}{4 \sqrt{2}}\left[-g e^{\frac{1}{\sqrt{2}} \phi}+3 m e^{-\frac{3}{\sqrt{2}} \phi}+\frac{2}{g} e^{-\frac{1}{\sqrt{2}} \phi}\left(k_{1} e^{-2 \lambda_{1}(r)}+k_{2} e^{-2 \lambda_{2}(r)}\right)\right]-\Upsilon e^{\frac{1}{\sqrt{2}} \phi-2 \lambda_{1}(r)-2 \lambda_{2}(r)}
\end{aligned}
$$

Introducing $x_{1}:=e^{2 \lambda_{1}-\sqrt{2} \phi}, x_{2}:=e^{2 \lambda_{2}-\sqrt{2} \phi}, u:=e^{2 \sqrt{2} \phi} x_{1} x_{2}=e^{2 \lambda_{1}+2 \lambda_{2}}$, we obtain the following flow equations, where we treat $x_{1}, x_{2}$ as a function of $u$.

$$
\begin{aligned}
& \frac{d x_{1}}{d u}=\frac{x_{1}}{u}\left[\frac{g^{3} m u-g^{2} m^{2} x_{1} x_{2}+2 g m\left(k_{1} x_{2}-k_{2} x_{1}\right)-4}{g^{3} m u+g^{2} m^{2} x_{1} x_{2}+2 g m\left(k_{1} x_{2}+k_{2} x_{1}\right)-4}\right], \\
& \frac{d x_{2}}{d u}=\frac{x_{2}}{u}\left[\frac{g^{3} m u-g^{2} m^{2} x_{1} x_{2}-2 g m\left(k_{1} x_{2}-k_{2} x_{1}\right)-4}{g^{3} m u+g^{2} m^{2} x_{1} x_{2}+2 g m\left(k_{1} x_{2}+k_{2} x_{1}\right)-4}\right] .
\end{aligned}
$$

We find there is only one supersymmetric fixed point where $x_{1}=x_{2}$, which we already know: $u=8 / g^{3} m, \quad x_{1}=x_{2}=2 / g m, \quad k_{1}=k_{2}=-1$.

One may try to construct series expansion solutions. From numerical solutions, we find that the solution aymptotes to $\mathrm{AdS}_{6}$ only if $k_{1}=k_{2}, x_{1}=x_{2}$. We thus do not present the series form of solutions here, since it should be identical with (3.8). For $k_{1}=-k_{2}$, we find there is a one-parameter 
family of numerical solutions connected to $\mathrm{AdS}_{6}$, and its series form is as follows.

$$
\begin{aligned}
& x_{1}=\sqrt{g /(3 m)} \sqrt{u}-\frac{2 k_{1}}{g m}+\sum_{n=1}^{\infty} \mathscr{C}_{n}^{(1)} u^{-n / 4}, \\
& x_{2}=\sqrt{g /(3 m)} \sqrt{u}-\frac{2 k_{2}}{g m}+\sum_{n=1}^{\infty} \mathscr{C}_{n}^{(2)} u^{-n / 4},
\end{aligned}
$$

where $\mathscr{C}_{1}^{(1)}=\mathscr{C}_{1}^{(2)}=\mathscr{C}_{1}$ is an integration constant, and the subleading coefficients can be found iteratively.

Below we report on the classification of IR singularities in general flows with $x_{1} \neq x_{2}$.

\begin{tabular}{|c|c|c|c|c|c|c|}
\hline$x_{1}$ & $x_{2}$ & $F$ & $e^{2 f}$ & $\left|g_{t t}^{10 d}\right|$ & $V(\phi)$ & Type \\
\hline$\infty$ & 0 & 0 & 0 & 0 & $\infty(\mathrm{Bad})$ & - \\
0 & $\infty$ & 0 & 0 & 0 & $\infty(\mathrm{Bad})$ & - \\
$\infty$ & $\infty$ & 0 & 0 & 0 & $\infty(\mathrm{Bad})$ & - \\
0 & 0 & 0 & $\infty$ & $\infty$ & $\infty(\mathrm{Bad})$ & $\mathrm{Bad}$ \\
\hline
\end{tabular}

\section{Lower-Dimensional Actions and Non-Supersymmetric Fixed Points}

It turns out that, upon application of partial twisting, $F(4)$ gauged supergravity allows various non-supersymmetric AdS solutions in addition to supersymmetric ones. They can be found either by solving the field equations in $D=6$ directly, or one can first work out a consistently truncated action in lower dimensions and look for critical points of the scalar potential thereof.

A simple approach in $D=6$ is to assume the existence of an AdS fixed point and write [29].

$$
e^{f}=\frac{\alpha}{g r} e^{-\frac{1}{\sqrt{2}} \phi}, \quad \quad e^{\lambda_{i}}=\frac{\beta_{i}}{g} e^{-\frac{1}{\sqrt{2}} \phi}, \quad \gamma=e^{-2 \sqrt{2} \phi},
$$

where $\alpha, \beta_{i}, \gamma$ are constants. We, then, obtain algebraic equations involving them, and, from their solutions, we have reproduced non-supersymmetric solutions with 2- and 3-cycles found in [29] and also discovered new non-supersymmetric solutions for 4-cycles, e.g. $A d S_{2} \times \mathscr{M}_{\mathrm{Kähler}}^{k=1}$ and $A d S_{2} \times$ $S^{2} \times S^{2}$ fixed points. We expect they can also be obtained as near horizon geometry of $A d S_{6}$ black holes whose horizon is $\mathscr{M}_{\mathrm{Kähler}}^{k=1}$ or $S^{2} \times S^{2}$. On the other hand, non-BPS $\mathrm{AdS}_{3}$ and $\mathrm{AdS}_{4}$ solutions correspond to near horizon geometry of black strings and black 2-branes, respectively.

\subsection{2- and 3- Cycles}

Let us start with the case of 2-cycles. From the field equations, we can of course doublecheck the supersymmetric solution with $k=-1, \alpha_{B P S}^{2}=8, \beta_{B P S}^{2}=4, \gamma_{B P S}=g /(2 m)$. There is in fact another solution which is non-supersymmetric [29], $\alpha_{\text {non-BPS }}^{2} \approx 6.61921, \beta_{\text {non-BPS }}^{2} \approx 3.47593$, $\gamma_{\text {non-BPS }} \approx 0.694146 \mathrm{~g} / \mathrm{m}$.

For 3-cycles, we reproduce a supersymmetric solution, $\alpha_{B P S}^{2}=9 / 2, \beta_{B P S}^{2}=3, \gamma_{B P S}=2 \mathrm{~g} / 3 \mathrm{~m}$, and also a non-supersymmetric one, at $\alpha_{\text {non-BPS }}^{2} \approx 5.27966, \beta_{\text {non-BPS }}^{2} \approx 3.41324$, and $\gamma_{\text {non-BPS }} \approx$ $0.507683 \mathrm{~g} / \mathrm{m}$. 


\subsubsection{Lower-dimensional action for 2- and 3-cycles}

One can straightforwardly check that by keeping only the modes $\lambda, \phi$ in BPS equations discussed earlier, and allowing general metric for the $(6-d)$-dimensional part, we obtain consistently truncated lower-dimensional actions. It can be also worked out collectively for $d=2$ and $d=3$. In Einstein-frame, the result is

$$
\begin{aligned}
\mathscr{S}_{6-d}^{\text {Ein }} & =\frac{\operatorname{Vol}\left(\mathscr{M}_{d}\right)}{2 \kappa_{6}^{2}} \int d^{6-d} x \sqrt{-g_{6-d}}\left[\frac{1}{4} R-\frac{d}{(4-d)} \partial_{\mu} \lambda \partial^{\mu} \lambda-\frac{1}{2} \partial_{\mu} \phi \partial^{\mu} \phi\right. \\
& \left.+\frac{k d}{4} e^{-\frac{8 \lambda}{4-d}}+\frac{1}{8} e^{-\frac{2 d \lambda}{4-d}}\left(g^{2} e^{\sqrt{2} \phi}+4 g m e^{-\sqrt{2} \phi}-m^{2} e^{-3 \sqrt{2} \phi}\right)-\frac{\tau_{\mathscr{M}_{d}}}{4 g^{2}} e^{-\frac{2(8-d) \lambda}{4-d}} e^{-\sqrt{2} \phi}\right],
\end{aligned}
$$

where $\tau_{\mathscr{M}_{d=2}}=2, \tau_{\mathscr{M}_{d=3}}=3 / 2$. We record that the metric ansatz which leads to the Einstein-frame action above is

$$
d s_{6}^{2}=e^{-\frac{2 d}{4-d} \lambda} d s_{6-d}^{2}+e^{2 \lambda} d s_{\mathscr{M}_{d}}^{2}
$$

\subsubsection{Stability of Non-Supersymmetric Solutions for 2- and 3-cycles}

The stability of supersymmetric solutions is guaranteed by unbroken supersymmetry, but, for non-supersymmetric solutions, there is no such guarantee. Thus we need to work out the eigenfrequency of fluctuation modes to check the stability. In this talk, we restrict ourselves to the modes kept by $D=6$ supergravity, which are the lightest modes and intuitively most likely to lead to tachyonic modes. We consider small fluctuations of $\lambda$ and $\phi$ around non-supersymmetric solutions of fields near non-supersymmetric AdS solutions, and diagonalize the mass matrix for $\lambda$ and $\phi$.

For 2-cycles, we find

$$
M_{\text {unstable }}^{2} R^{2} \approx-3.032 \leq-\frac{9}{4}, \quad M_{\text {stable }}^{2} R^{2} \approx 1.741 \geq-\frac{9}{4},
$$

where $\mathrm{BF}$ bound for $\mathrm{AdS}_{4}$ is $M_{\text {scalar }}^{2} R^{2} \geq-\frac{9}{4}$, so we conclude this solution is unstable.

For 3 cycles, we obtain

$$
M_{\text {unstable }}^{2} R^{2} \approx-1.593 \leq-1, \quad M_{\text {stable }}^{2} R^{2} \approx-0.444 \geq-1,
$$

where $\mathrm{BF}$ bound for $\mathrm{AdS}_{3}$ is $M_{\mathrm{scalar}}^{2} R^{2} \geq-1$, so we again encounter instability.

\subsection{4-Cycles}

One can verify the BPS solutions for negatively curved 4-cycles and also find non-BPS solutions for positively curved Kähler 4-cycles which are locally $S^{2} \times S^{2}$ or $\mathbb{C P}^{2}$.

\subsubsection{Fixed Point Solutions for Cayley and Kähler 4-Cycles}

For Cayley cycles, it turns out that there are no AdS solutions other than the BPS solution: $\alpha_{B P S}^{2}=2, \beta_{B P S}^{2}=8 / 3$, and $\gamma_{B P S}=3 g /(4 m)$. 
For Kähler cycles on the other hand, we find, in addition to a supersymmetric solution with $k=-1, \alpha_{B P S}^{2}=2, \beta_{B P S}^{2}=4$, and $\gamma_{B P S}=g /(2 m)$, there is a non-BPS solutions for $k=1$, having

$$
\alpha_{n o n-B P S}^{2}=\frac{1}{5}(4-\sqrt{6}), \quad \beta_{n o n-B P S}^{2}=\frac{4}{5}(4-\sqrt{6}), \quad \gamma_{n o n-B P S}=\frac{1}{4}(2+\sqrt{6}) \frac{g}{m} .
$$

Although there could be solutions with different scalar curvature and radius for two Riemann surfaces, we find there is no additional fixed point than reported already in previous subsections.

\subsubsection{Two Dimensional Theories on 4-Cycles}

We here present the bosonic action for two dimensional effective theories on $\mathscr{M}_{4}$, which can be a supersymmetric four-cycle, i.e. Cayley or Kähler. As it is well known, one cannot move to Einstein frame through scale transformation in 2 dimensions and that is why there is a conformal factor $e^{\lambda_{1}+\lambda_{2}}$ below.

$$
\begin{aligned}
\mathscr{S}_{2}= & \frac{\operatorname{Vol}\left(\mathscr{M}_{4}\right)}{2 \kappa_{6}^{2}} \int d^{2} x \sqrt{-g_{2}} e^{2 \lambda_{1}+2 \lambda_{2}}\left[\frac{1}{4} R_{2}+\frac{1}{2}\left(e^{-2 \lambda_{1}} k_{1}+e^{-2 \lambda_{2}} k_{2}\right)\right. \\
& +\frac{1}{2} g^{\mu v} \partial_{\mu} \lambda_{1} \partial_{v} \lambda_{1}+\frac{1}{2} g^{\mu v} \partial_{\mu} \lambda_{2} \partial_{v} \lambda_{2}+2 g^{\mu v} \partial_{\mu} \lambda_{1} \partial_{v} \lambda_{2}-\frac{1}{2} \partial_{\mu} \phi \partial^{\mu} \phi \\
& +\frac{1}{8}\left(g^{2} e^{\sqrt{2} \phi}+4 g m e^{-\sqrt{2} \phi}-m^{2} e^{-3 \sqrt{2} \phi}\right) \\
& \left.-\frac{\tau_{\mathscr{M}_{4}}}{4 g^{2}} e^{-\sqrt{2} \phi}\left(e^{-4 \lambda_{1}}+e^{-4 \lambda_{2}}\right)-\frac{\tau_{\mathscr{M}_{4}}^{2}}{2 m^{2} g^{4}} e^{\sqrt{2} \phi-4 \lambda_{1}-4 \lambda_{2}}\right],
\end{aligned}
$$

where $\tau_{\mathscr{M}_{\text {Cayley }}}=2 / 3, \tau_{\mathscr{M}_{\text {Käler }}}=2$, and $\tau_{\Sigma_{1} \times \Sigma_{2}}=2$. Note that for Cayley and Kähler 4-cycles as e.g. $\mathbb{C P}^{2}$ we need to set $\lambda_{1}=\lambda_{2}$ and $k_{1}=k_{2}$. From this effective action, one can reproduce all the results above involving 4-cycles. We record the reduction ansatz for $D=6$ metric is

$$
d s_{6}^{2}=d s_{2}^{2}+\sum_{i=1}^{2} e^{2 \lambda_{i}} d s_{\mathscr{M}_{i}}^{2} .
$$

From the action and the equations of motion, we have calculated the mass eigenvalues of scalar fluctuations around the non-supersymmetric $A d S_{2} \times S^{2} \times S^{2}$ and the result is

$$
M_{1}^{2} R^{2}=\frac{3}{20}(6+\sqrt{6}), \quad M_{2}^{2} R^{2}=3, \quad M_{3}^{2} R^{2}=\frac{1}{4}(6+\sqrt{6}) .
$$

We thus find there is no unstable mode.

\section{Discussions}

In this talk, we have analyzed all fixed points and holographic renormalization group flows ${ }^{5}$ associated with the geometries which describe the branes wrapping on calibrated cycles in several special holonomy manifolds with appropriate topological twists. We have also tried to determine if the IR singularities are physically admissible, but, for some cases, the Maldacena-Nuñez criterion

\footnotetext{
${ }^{5}$ More discussions on two dimensional solutions are now available in [39] and references therein.
} 
and the Gubser criterion give us contradictory answers. We thus need to perform more elaborate analysis such as the construction of black hole solutions where the singularity is hidden behind the horizon. We postpone this work to future works.

In addition, we have also worked out lower-dimensional consistently truncated action in 4, 3, and 2 dimensions. Using them, we have checked the stability of the non-supersymmetric solutions with respect to the Breitenlohner-Freedman bound. Let us emphasize that the lower dimensional actions we have presented are not yet the bosonic part of some supersymmetric action. We need to consider vector and tensor fields, additionally, in the same way as [40, 41, 42]. One might be able to find the interesting new solutions, e.g. exhibiting Lifshitz-scaling [40], and we postpone this problem also to future works.

From the viewpoint of recent developments concerning the comparison using AdS/CFT, we point out that there exist gravity solutions whose field theory dual is not amenable to localization treatment. It is mainly due to insufficient amount of preserved supersymmetry. For instance, the $\mathrm{AdS}_{2}$ solution wrapped on Cayley 4-cycle has only two supercharges, and we do not know how to do the field theory side calculation. It is similar to the situation with sphere partition functions: we need extended supersymmetry, i.e. $\mathscr{N}=2$ (8 supercharges) is needed to put the theory on $S^{4}$ and localize [43], and similarly to put a three-dimensional theory on $S^{3}$ and localize one needs $\mathscr{N}=2$ (4 supercharges) [44, 45]. Our final comment is that a number of supergravity solutions are still waiting for field theory computation to catch up.

\section{Acknowledgments}

We are grateful to the organizers of Corfu Summer Institute 2019 for the hospitality and the chance to give this talk during the conferences. This work was supported by the National Research Foundation (NRF) grant 2018R1D1A1B07045414. MS is also supported by a scholarship from Hyundai Motor Chung Mong-Koo Foundation.

\section{References}

[1] N. Kim and M. Shim, Wrapped Brane Solutions in Romans F(4) Gauged Supergravity, Nucl. Phys. B951 (2020) 114882 [1909.01534].

[2] J. M. Maldacena, The Large N limit of superconformal field theories and supergravity, Int. J. Theor. Phys. 38 (1999) 1113 [hep-th/9711200].

[3] J. P. Gauntlett, J. B. Gutowski, C. M. Hull, S. Pakis and H. S. Reall, All supersymmetric solutions of minimal supergravity in five- dimensions, Class. Quant. Grav. 20 (2003) 4587 [hep-th/ 0209114 ].

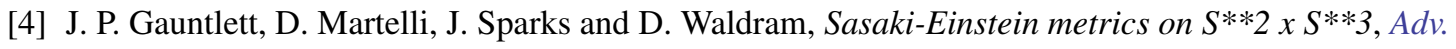
Theor. Math. Phys. 8 (2004) 711 [hep-th/ 0403002 ].

[5] N. Kim, AdS(3) solutions of IIB supergravity from D3-branes, JHEP 01 (2006) 094 [hep-th/0511029].

[6] N. Kim and J.-D. Park, Comments on AdS(2) solutions of D=11 supergravity, JHEP 09 (2006) 041 [hep-th/0607093].

[7] J. P. Gauntlett, N. Kim and D. Waldram, Supersymmetric AdS(3), AdS(2) and Bubble Solutions, JHEP 04 (2007) 005 [hep-th/ 0612253 ]. 
[8] J. P. Gauntlett and N. Kim, Geometries with Killing Spinors and Supersymmetric AdS Solutions, Commun. Math. Phys. 284 (2008) 897 [0710.2590].

[9] A. Donos, J. P. Gauntlett and N. Kim, AdS Solutions Through Transgression, JHEP 09 (2008) 021 [0807.4375].

[10] M. Pernici and E. Sezgin, Spontaneous Compactification of Seven-dimensional Supergravity Theories, Class. Quant. Grav. 2 (1985) 673.

[11] L. J. Romans, The F(4) Gauged Supergravity in Six-dimensions, Nucl. Phys. B269 (1986) 691.

[12] M. Cvetic, H. Lu and C. N. Pope, Gauged six-dimensional supergravity from massive type iia, hep-th/9906221v1.

[13] J. Jeong, O. Kelekci and E. Ó Colgáin, An alternative IIB embedding of $F(4)$ gauged supergravity, JHEP 05 (2013) 079 [1302.2105].

[14] J. Hong, J. T. Liu and D. R. Mayerson, Gauged Six-Dimensional Supergravity from Warped IIB Reductions, JHEP 09 (2018) 140 [1808.04301].

[15] E. Malek, H. Samtleben and V. V. Camell, Supersymmetric ads ${ }_{7}$ and ads 6 vacua and their consistent truncations with vector multiplets, http: / / arxiv.org/abs/1901.11039v1.

[16] N. Seiberg, Five-dimensional SUSY field theories, nontrivial fixed points and string dynamics, Phys. Lett. B388 (1996) 753 [hep-th/9608111].

[17] D. R. Morrison and N. Seiberg, Extremal transitions and five-dimensional supersymmetric field theories, Nucl. Phys. B483 (1997) 229 [hep-th/ 9609070$].$

[18] K. A. Intriligator, D. R. Morrison and N. Seiberg, Five-dimensional supersymmetric gauge theories and degenerations of Calabi-Yau spaces, Nucl. Phys. B497 (1997) 56 [hep-th/9702198].

[19] A. Brandhuber and Y. Oz, The D-4 - D-8 brane system and five-dimensional fixed points, Phys. Lett. B460 (1999) 307 [hep-th/ 9905148$].$

[20] D. L. Jafferis and S. S. Pufu, Exact results for five-dimensional superconformal field theories with gravity duals, JHEP 05 (2014) 032 [1207 . 4359].

[21] F. Benini and A. Zaffaroni, A topologically twisted index for three-dimensional supersymmetric theories, JHEP 07 (2015) 127 [1504.03698].

[22] F. Benini, K. Hristov and A. Zaffaroni, Black hole microstates in AdS $S_{4}$ from supersymmetric localization, JHEP 05 (2016) 054 [1511. 04085 ].

[23] S. M. Hosseini, I. Yaakov and A. Zaffaroni, Topologically twisted indices in five dimensions and holography, JHEP 11 (2018) 119 [1808.06626].

[24] S. M. Hosseini, K. Hristov, A. Passias and A. Zaffaroni, 6D attractors and black hole microstates, 1809.10685 .

[25] P. M. Crichigno, D. Jain and B. Willett, 5d Partition Functions with A Twist, JHEP 11 (2018) 058 [1808.06744].

[26] M. Suh, Supersymmetric AdS 6 black holes from F(4) gauged supergravity, JHEP 01 (2019) 035 [1809.03517].

[27] M. Suh, Supersymmetric AdS $S_{6}$ black holes from matter coupled F(4) gauged supergravity, JHEP 02 (2019) 108 [1810.00675]. 
[28] M. Suh, On-shell action and the Bekenstein-Hawking entropy of supersymmetric black holes in AdS , 1812.10491.

[29] M. Naka, Various wrapped branes from gauged supergravities, hep-th/0206141.

[30] J. M. Maldacena and C. Nuñez, Supergravity description of field theories on curved manifolds and a no go theorem, Int. J. Mod. Phys. A16 (2001) 822 [hep-th/ 0007018 ].

[31] S. S. Gubser, Curvature singularities: The Good, the bad, and the naked, Adv. Theor. Math. Phys. 4 (2000) 679 [hep-th/0002160].

[32] J. P. Gauntlett, N. Kim, S. Pakis and D. Waldram, M theory solutions with AdS factors, Class. Quant. Grav. 19 (2002) 3927 [hep-th/ 0202184 ].

[33] P. Breitenlohner and D. Z. Freedman, Stability in Gauged Extended Supergravity, Annals Phys. 144 (1982) 249.

[34] E. Malek, H. Samtleben and V. Vall Camell, Supersymmetric $A d S_{7}$ and $A d S_{6}$ vacua and their minimal consistent truncations from exceptional field theory, Phys. Lett. B786 (2018) 171 [1808 . 05597 ].

[35] J. M. Maldacena and C. Nuñez, Towards the large N limit of pure N=1 superYang-Mills, Phys. Rev. Lett. 86 (2001) 588 [hep-th/ 0008001$].$

[36] B. S. Acharya, J. P. Gauntlett and N. Kim, Five-branes wrapped on associative three cycles, Phys. Rev. D63 (2001) 106003 [hep-th/ 0011190 ].

[37] J. P. Gauntlett, N. Kim and D. Waldram, M Five-branes wrapped on supersymmetric cycles, Phys. Rev. D63 (2001) 126001 [hep-th/ 0012195 ].

[38] J. P. Gauntlett and N. Kim, M five-branes wrapped on supersymmetric cycles. 2., Phys. Rev. D65 (2002) 086003 [hep-th/0109039].

[39] M. Suh, Holographic renormalization group flows in two-dimensional gravity and AdS black holes, 2002.07194.

[40] A. Donos, J. P. Gauntlett, N. Kim and O. Varela, Wrapped M5-branes, consistent truncations and AdS/CMT, JHEP 12 (2010) 003 [1009.3805].

[41] E. Ó Colgáin and H. Samtleben, 3D gauged supergravity from wrapped M5-branes with AdS/CMT applications, JHEP 02 (2011) 031 [1012.2145].

[42] K. C. M. Cheung, J. P. Gauntlett and C. Rosen, Consistent KK truncations for M5-branes wrapped on Riemann surfaces, 1906.08900.

[43] V. Pestun, Localization of gauge theory on a four-sphere and supersymmetric Wilson loops, Commun. Math. Phys. 313 (2012) 71 [0712.2824].

[44] D. L. Jafferis, The Exact Superconformal R-Symmetry Extremizes Z, JHEP 05 (2012) 159 [1012.3210].

[45] N. Hama, K. Hosomichi and S. Lee, Notes on SUSY Gauge Theories on Three-Sphere, JHEP 03 (2011) 127 [1012.3512]. 